\title{
SALDI-MS Signal Enhancement Using Oxidized Graphitized Carbon Black Nanoparticles
}

\author{
Nahid Amini, ${ }^{*}$ Mohammadreza Shariatgorji, ${ }^{*}$ and Gunnar Thorsén \\ Department of Analytical Chemistry, Stockholm University, Stockholm, Sweden
}

\begin{abstract}
The signal intensity of low-molecular-weight compounds analyzed using surface-assisted laser desorption/ionization time-of-flight mass spectrometry (SALDI-TOF-MS) was significantly enhanced when oxidized graphitized carbon black (GCB) particles were used as the desorption/ionization surface. The surface of oxidized GCB contains more carboxylic acid groups than non-oxidized GCB. Carboxylic acid groups enhance the efficiency of the ionization process and the desorption of more hydrophobic compounds. A common pharmaceutical compound, propranolol, was successfully extracted from Baltic Sea blue mussels and quantified using oxidized GCB as the SALDI surface, whereas deuterated propranolol was used as the internal standard. The calibration curve showed a wide linear dynamic range of response $(0.1-20 \mu \mathrm{g} / \mathrm{mL})$ and good reproducibility (RSD $<10 \%$ ). It was not possible to detect propranolol in Baltic Sea blue mussels when non-oxidized GCB was used as the SALDI surface. (J Am Soc Mass Spectrom 2009, 20, 1207-1213) (c) 2009 American Society for Mass Spectrometry
\end{abstract}

$\mathrm{D}$ espite all the efforts made in overcoming problems associated with analysis of small molecules using matrix-assisted laser desorption ionization mass spectrometry (MALDI-MS) [1, 2], the technique still requires further improvements. A range of techniques has been developed to overcome the matrix problems in analysis of small molecules, including the use of desorption ionization from porous silicon (DIOS) [3], polymeric matrices [4], inorganic materials [5], high-molecular-weight matrices [6], surfactantsuppressed matrices [7], and carbon allotropes. Carboneous materials such as carbon nanotubes [8], activated carbon [9], graphite [10], and, recently, graphitized carbon black (GCB) [11] have been shown to provide suitable media for surface-assisted laser desorption ionization (SALDI) and have been used in various formats for different applications. Among these techniques DIOS is the most widely used, although it suffers from oxidation of surface groups and degradation of performance when stored in air for extended periods of time [12].

Several strategies exist to reduce the detection limits of desorption/ionization MS analysis. One approach to improve sensitivity is to concentrate the sample on a plate, as in surface-enhanced laser desorption ionization (SELDI) [13], or on a medium that can act as a SALDI matrix. Graphitized carbon black has been re-

Address reprints requests to Dr. Gunnar Erik Thorsén, Stockholm University, Department of Analytical Chemistry, Svante Arrhenius v 12, S-10691 Stockholm, Sweden. E-mail: gunnar.thorsen@anchem.su.se

* These authors contributed equally to this work. cently used as a bifunctional material to concentrate small molecules (as a solid-phase extraction sorbent) and to act as a SALDI medium [11]. Another approach is to enhance the desorption/ionization efficiency by increasing the number of cation-exchangeable sites on the surface [8], such as through oxidation.

The formation of surface oxides using different liquid oxidizing agents in a variety of carbonaceous materials has been summarized in several reports [14-16], but no report is available on the oxidation of GCB. GCB, which is obtained by heating carbon black at $2700-$ $300{ }^{\circ} \mathrm{C}$, has a relatively small proportion of residual oxygen on the surface, mainly as a result of contamination of the particles before graphitization [17]. To increase the amount of chemisorbed oxygen on the surface, a concentrated $\mathrm{H}_{2} \mathrm{SO}_{4} / \mathrm{HNO}_{3}$ mixture was used, based on findings from carbon nanofibers [16].

One advantage of GCB materials over carbon nanotubes, which have been used as a SALDI matrix in their oxidized form [8], is their low cost. The yield of the oxidation reaction is low at roughly $15 \%$ [8] and analytical protocols using less expensive materials need to be developed.

Human pharmaceuticals have recently attracted attention as emerging environmental contaminants [18]. The occurrence of pharmaceuticals in the environment is well documented [19], although little is known about their environmental fate and availability [20] or their uptake, possible accumulation, and effects in biota [21]. The determination of the human pharmaceutical propranolol in Baltic Sea blue mussels (Mytilus edulus), exposed to the drug as part of a sublethal chronic 
exposure experiment, was chosen for an evaluation of the performance of quantitative SALDI analysis.

\section{Experimental}

\section{Materials and Reagents}

Graphitized carbon black 4 (GCB 4; mesh $>400$, surface area $210 \mathrm{~m}^{2} / \mathrm{g}$ ) was kindly provided by the Laboratori Analitici di Ricerca Associati (Formello, Rome, Italy). Tetramethylammonium bromide and tetraethylammonium bromide were purchased from Fluka Chemie (Buchs, Switzerland), tetrapropylammonium bromide and tetrapentylammonium bromide from Aldrich (Milwaukee, WI, USA), and tetrabutylammonium bromide from Merck (Darmstadt, Germany). The organophosphate esters tetraethyl ethylenediphosphonate, triphenyl phosphate, tris(n-butyl) phosphate, tris(2-butoxyethyl) phosphate, tritolyl phosphate, tris (n-pentyl) phosphate, tris(n-propyl) phosphate, and the aminoglycoside antibiotics gentamycine, neomycine, dihydroxystreptomycine, streptomycine, and tobramycine were obtained from Sigma-Aldrich (St. Louis, MO, USA), as was trifluoroacetic acid (TFA). Sulfathiazole, sulfanilamide, sulfaquinoxaline, sulfaphenazole, sulfadimethoxine, sulfisomidine, sulfadiazine, sulfamonomethoxine, sulfamethoxazole, acebutolol, metoprolol, propranolol, carbamazepine, naproxen, ibuprofen, and clenbuterol were purchased from Sigma. Deuterated propranolol (ring-d7) was obtained from QMX Laboratories (Essex, UK). Hydrochloric acid, sulfuric acid, nitric acid, $n$-hexane, acetonitrile, ethyl acetate, ammonia, dichloromethane, and sodium carbonate were purchased from Merck. Water was purified using a Millipore water purification system (Millipore Corp., Billerica, MA, USA) to a resistance $>18 \mathrm{M} \Omega / \mathrm{cm}$.

\section{Procedure for Oxidation of GCB}

A mixture of GCB (300 mg) and concentrated $\mathrm{HNO}_{3}$ / $\mathrm{H}_{2} \mathrm{SO}_{4}(40 \mathrm{~mL}, 1: 3$, vol/vol) was stirred and refluxed at $120^{\circ} \mathrm{C}$ for $30 \mathrm{~min}$ [8]. The solution was cooled to room temperature, diluted to $200 \mathrm{~mL}$ with water, transferred into 15-mL Falcon conical tubes (BD Biosciences, Franklin Lakes, NJ, USA), and centrifuged (Eppendorf 5415 R, $9659 \times g)$. The supernatant liquid from each tube was discarded, more water was added, and the mixture was centrifuged again. This process was repeated seven times until the supernatant water became neutral. The final precipitate was separated and allowed to dry at room temperature. Approximately $30 \mathrm{mg}$ of oxidized GCB was collected and stored under dry conditions for further use.

\section{Characterization of Oxidized GCB}

SEM. The elemental analyses of GCB and oxidized GCB were performed on a field emission scanning microscope (JSM-7000F, JEOL, Tokyo, Japan) with wavelength and energy dispersive spectroscopy (WDS and EDS), on four different spots.

Titration. GCB and oxidized GCB samples were washed with water and allowed to dry at ambient temperature. The samples were then heated at $100{ }^{\circ} \mathrm{C}$ for $5 \mathrm{~h}$ before titration. A titration procedure was conducted in triplicate to quantify the carboxylic acid groups present in the oxidized GCB [22]. Oxidized GCB was stirred in $2.00 \mathrm{~mL}$ of $0.05 \mathrm{~N} \mathrm{NaHCO}_{3}$ aqueous solution for $48 \mathrm{~h}$. Then $2.00 \mathrm{~mL}$ of $0.05 \mathrm{~N}$ aqueous $\mathrm{HCl}$ was added to the solution, which was boiled for $5 \mathrm{~min}$ to remove any $\mathrm{CO}_{2}$ present. The solution was cooled to room temperature and the excess $\mathrm{HCl}$ was titrated with $0.05 \mathrm{~N}$ aqueous $\mathrm{NaOH}$ until pH 7.00 was reached (Jenway $\mathrm{pH}$ meter, Essex, UK). A volume equal to $156 \mu \mathrm{L}$ of titrand was added to $2.79 \mathrm{mg}$ of oxidized GCB to achieve this.

NMR. Proton spectra were recorded on a Bruker Avance spectrometer (Bruker BioSpin GmbH, Rheinstetten, Germany) operating at 9.4 Tesla, using an inverse-detection (BBI, Bruker BioSpin) probe. The sample was once dispersed in deuterated water $\left(\mathrm{D}_{2} \mathrm{O}\right)$ and water-suppression techniques were used, by excitation sculpting with gradients. For the sample with dimethylsulfoxide (DMSO) as solvent, a normal $90^{\circ}$ pulse sequence was used. In all, 256 scans were acquired using the hard ${ }^{1} \mathrm{H} \pi / 2$ pulse of $8 \mu$ s at room temperature.

\section{Sample Preparation and Spotting on a Plate}

Five groups of compounds consisting of human medicines, sulfonamides, organophosphate esters, aminoglycoside antibiotics, and quaternary amines were selected. Solutions with concentrations of $0.07 \mathrm{mM}$ for each group were prepared and stored at around $4{ }^{\circ} \mathrm{C}$ before use.

GCB $(0.1 \mathrm{~g})$ was washed with $1 \mathrm{~mL}$ of $\mathrm{MeOH}$ and 1 $\mathrm{mL}$ of $\mathrm{CH}_{2} \mathrm{Cl}_{2} / \mathrm{MeOH} 80 / 20$ using a 3-mL fritted reservoir (Isolute; Biotage, Charlottesville, UK) mounted on a solid-phase extraction vacuum manifold (VacMaster10, Biotage). Nitrogen was then passed through the reservoir to dry the particles. After reaching dryness, a suspension containing $10 \mathrm{mg}$ of GCB in $1 \mathrm{~mL}$ of water was prepared. The suspension was vigorously shaken and $1.5 \mu \mathrm{L}$ was spotted on a stainless steel plate. Oxidized GCB (10 mg) was dispersed into a suspension in $1 \mathrm{~mL}$ of water, resulting in a very uniform and stable suspension, and $1.5 \mu \mathrm{L}$ of the suspension was spotted on a stainless steel plate. Spots were left to dry, after which $1.5 \mu \mathrm{L}$ of the sample solutions were 
added to each spot together with $1 \mu \mathrm{L}$ of $0.1 \%$ TFA. When completely dry, the plates were stored at room temperature awaiting laser desorption/ionization analysis.

\section{Extraction and Quantification of Propranolol from Mussel Tissue}

For the exposure experiments, the mussels were placed in aquaria filled with artificial sea water containing different concentrations of propranolol. The aquaria were kept at $7{ }^{\circ} \mathrm{C}$ throughout the exposure experiment and all of them were aerated. The exposure experiment was conducted over 14 days. Every third day the water was exchanged in the experimental jars, food supplied (I. galbana $\sim 0.9 \mathrm{mg} \mathrm{dw} \mathrm{L}^{-1}$ ), and propranolol was added. At the end of the 14 days, the mussels were subjected to a scope for growth experiment [23]. In this experiment a total energy budget was calculated for the blue mussel, based on assimilation, excretion, and respiration, and this can be used to measure the state of health or the degree of stress of an organism. The mussels were frozen immediately after the scope for growth experiment and kept at $-18^{\circ} \mathrm{C}$ until chemical analysis was performed.

Frozen mussels were thawed, homogenized, and weighed. To each sample, which weighed about $30 \mathrm{mg}$, $2 \mathrm{~mL}$ of acetonitrile was added, mixed vigorously using a Vortex mixer (Whirlmixer, Fisons, UK), and centrifuged $(6036 \times g$; Damon/IEC Division, Foster City, CA, USA). The extraction procedure was repeated three times. Subsequently, $1 \mathrm{~mL}$ of $0.1 \mathrm{M} \mathrm{HCl}$ and $1 \mathrm{~mL}$ of $n$-hexane were added to the acetonitrile extract, mixed, centrifuged, and the $n$-hexane supernatant was separated and discarded. This procedure was repeated three more times but without the addition of acid. The acetonitrile phase, containing propranolol, was evaporated to a few milliliters in a vacuum using a rotary evaporator (SpeedVac, Savant SPD111V, Thermo Fisher Scientific, Waltham, MA, USA), transferred to a centrifuge tube, and $1 \mathrm{~mL}$ of $0.1 \mathrm{M} \mathrm{HCl}$ and $1 \mathrm{~mL}$ of ethyl acetate were added to it. The mixture was shaken and centrifuged after each of three additions of ethyl acetate. The ethyl acetate supernatants were collected, combined, and evaporated to dryness under a stream of nitrogen and the dry residues were dissolved in $50 \mu \mathrm{L}$ of $1 \%$ TFA in water. The water content of mussels, measured after reaching a constant weight at $80^{\circ} \mathrm{C}$, was roughly $97 \%$.

Deuterated propranolol was used as the internal standard and was added to the standard solutions used to construct the calibration curve $(0.1,0.5,1,5,10,15$, and $20 \mu \mathrm{g} / \mathrm{mL}$ ) and to the mussel samples at a concentration of $1.0 \mu \mathrm{g} / \mathrm{mL}$.

To investigate the extraction yield of propranolol from mussels, $25 \mu \mathrm{L}$ of a $20 \mu \mathrm{g} / \mathrm{mL}$ solution of propranolol was added to four samples of blank mussels and extraction was performed as stated earlier. Using deuterated propranolol as the internal standard, the extracted propranolol was quantified using the calibration curve.

\section{SALDI-ToF-MS}

All SALDI mass spectra were obtained using a Voyager DE-STR time-of-flight mass spectrometer (Applied Biosystems, Carlsbad, CA, USA), in the reflector mode. A pulsed 337-nm nitrogen laser was used for desorption. The acceleration voltage was $20 \mathrm{kV}$, the delay time was $150 \mathrm{~ns}$, and the grid voltage was $65 \%$. All mass spectra were accumulated from five different spots, 20 shots per spot, except for the quaternary amine samples in which the final spectrum was the average of five different spots and five shots per spot. The signal-to-noise $(\mathrm{S} / \mathrm{N})$ values were calculated using the automatic $\mathrm{S} / \mathrm{N}$ calculator in Data Explorer version 4.0. The laser intensity was optimized to give as wide dynamic range as possible for the experiments with the quaternary amines. The laser intensity was kept constant for the quantitative measurements as well as for all experiments with comparisons between GCB and oxidized GCB.

\section{Results and Discussion}

\section{Characterization of Oxidized GCB 4}

The scanning electron microscope (SEM) images (Figure 1) show that the particles of GCB have been reduced to $<100 \mathrm{~nm}$ in size after oxidation. This observation is in agreement with findings showing that a concentrated $\mathrm{H}_{2} \mathrm{SO}_{4} / \mathrm{HNO}_{3}$ mixture cuts carbon nanotubes [8] and nanofibers [16] into shorter lengths. The particles of oxidized GCB have a tendency to aggregate, as shown in Figure 1b. Elemental analysis was performed using SEM and the oxygen content of GCB was found to be $5.5 \pm 0.1 \%(\mathrm{wt} / \mathrm{wt})$, whereas oxidized GCB contained $14.1 \pm 1.3 \%(\mathrm{wt} / \mathrm{wt})$ oxygen. The results revealed that oxidized GCB had higher oxygen content than that of GCB but they did not provide any information on the nature of the oxygenated functional groups present.

To evaluate the percentage of oxygen present as $\mathrm{COOH}$, acid-base titration was performed. GCB exhibited basic properties ( $\mathrm{pH} 8.1$ at $3 \mathrm{mg} / \mathrm{mL}$ of GCB), which is in agreement with previous observations [17]. The $\mathrm{pH}$ value for $3 \mathrm{mg} / \mathrm{mL}$ solutions of oxidized GCB was roughly 2.9, which corresponded to the presence of $12.2 \pm 1.0 \%$ (wt/wt) $\mathrm{COOH}$, equivalent to $8.7 \pm 0.7 \%$ (wt/wt) oxygen. From SEM and titration experiments it can be concluded that $5.4 \pm 1.5 \%(w t / w t)$ of the oxygen in oxidized GCB comprises functional groups other than $\mathrm{COOH}$. The presence of $\mathrm{COOH}$ groups on oxidized GCB was further confirmed by nuclear magnetic resonance (NMR) imaging.

NMR studies of functionalized nanoparticles, such as carbon nanotubes, have attracted attention because of 

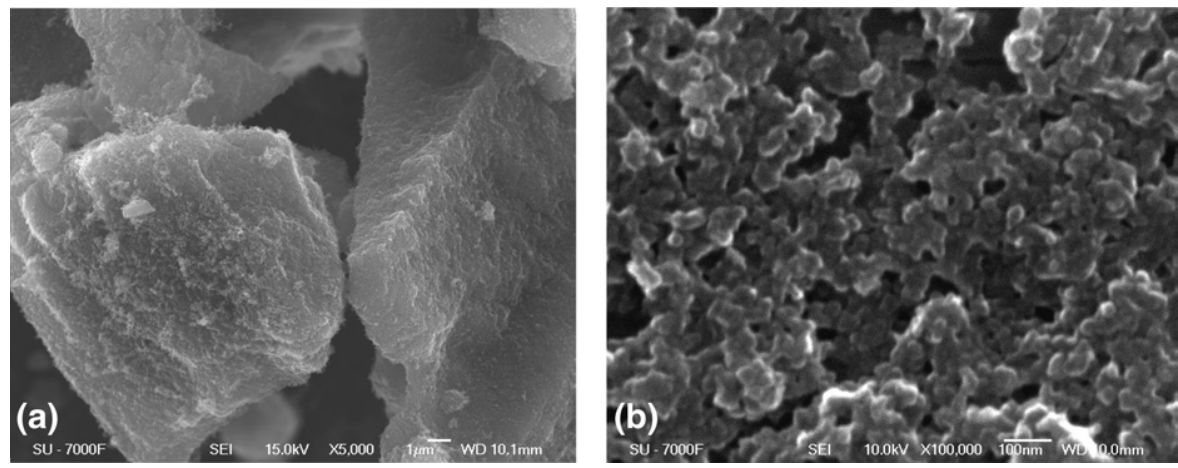

Figure 1. SEM images of $(\mathbf{a})$ GCB $(\times 5000)$ and $(\mathbf{b})$ oxidized GCB $(\times 100,000)$. The particle size of GCB has reduced by a factor of about $10^{5}$ after oxidation.

their potential to probe the functional groups and surface characterization. Although solid-state NMR is the technique of choice, liquid state NMR is also informative when the material of interest is soluble [24]. Two NMR proton spectra were taken, one in DMSO and the other in $\mathrm{D}_{2} \mathrm{O}$. A sharp proton signal appeared at $8 \mathrm{ppm}$ in both cases, whereas a broader signal (at $13.8 \mathrm{ppm}$ ) was observed only in DMSO. The signal observed at 8 ppm was not exchangeable, whereas the signal at 13.8 ppm was exchanged with the deuterium present in $\mathrm{D}_{2} \mathrm{O}$. The signal at $13.8 \mathrm{ppm}$, which probably corresponds to carboxylic protons, was notably shifted to the low-field region. This could be attributed to the extent of hydrogen bonding between the surface-bound carboxylic groups $[25,26]$. The acidic properties of the carboxylic groups could also be amplified by electron delocalization over the large network of aromatic rings in the graphene structure. The signal at $8 \mathrm{ppm}$ corresponds to aromatic protons present at the edges of the graphene structure.

\section{Comparison of Ionization/Desorption Efficiencies of the GCB and Oxidized GCB}

Experiments were performed using a number of quaternary amines (QAs) as model compounds to study how the differences in surface characteristics between GCB and oxidized GCB influence the desorption process. When the QAs were used, desorption could be studied independently of the ionization efficiency of the analyte molecules. The selected QAs, with alkyl chains increasing long from methyl to pentyl, differed in their hydrophobic properties.

The $\mathrm{S} / \mathrm{N}$ ratio was determined for different QAs to investigate the hypothesis that the addition of oxygencontaining groups to GCB not only improves the ionization efficiency by providing enough cations but also substantially changes the nature of the SALDI surface, thus creating different desorption behaviors.

Figure 2 presents the $\mathrm{S} / \mathrm{N}$ ratios obtained for QAs on the surface of GCB and oxidized GCB. The relative desorption efficiencies of QAs with different alkyl chain lengths were reversed from GCB to oxidized GCB. Less hydrophobic QAs were more easily desorbed from the more hydrophobic surface of GCB, whereas the hydrophilic surface of oxidized GCB readily desorbed the more hydrophobic QAs. The QA desorption profiles suggest that the hydrophobic surface of GCB has been changed by oxidation. Thus the improvement in $\mathrm{S} / \mathrm{N}$ ratios using oxidized GCB should be attributed to both improvement in the ionization of the target compounds and to changes in the desorption process. From a theoretical perspective the intermolecular forces (such as hydrophobic interactions, dispersion forces, London forces) between the SALDI surface and the analyte seem to inhibit desorption.

\section{Enhancement of SALDI-MS Signal-to-Noise Ratios}

The characterization of the oxidized particles by NMR and titration showed a substantial increase in the number of acidic functional groups present on the particle surface, whereas SEM confirmed the increase in the number of oxygen. These groups can act as cationdonor sites by providing adsorbed analytes with protons, sodium ions, or potassium ions, increasing the

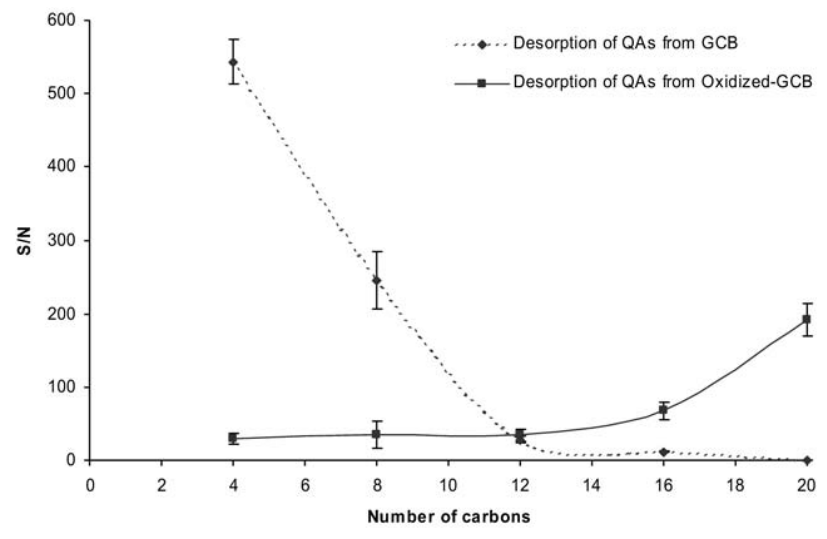

Figure 2. The desorption patterns of QAs from both GCB and oxidized GCB surfaces. The less hydrophobic QAs were more easily desorbed from the surface of GCB, whereas oxidized GCB was the preferred desorption surface for the more hydrophobic QAs. 
signal intensities of compounds in positive-mode SALDI-MS. Four groups of model compounds were selected: human medicines, sulfonamides, organophosphate esters, and aminoglycoside antibiotics. The compounds in the selected groups covered a wide range of physical and chemical properties to evaluate the ionization/desorption efficiency of the nanoparticles. Figure 3 shows the $\mathrm{S} / \mathrm{N}$ ratios of proton, sodium, and potassium adducts of a selected number of the compounds studied obtained using both GCB and oxidized GCB nanoparticles. The complete list of compounds including the relative standard deviation (RSD) value for each adduct is presented in the supplementary material, which can be found in the electronic version of this article. Most of the results demonstrate a significant increase in the $\mathrm{S} / \mathrm{N}$ ratio, particularly for basic compounds. Dihydroxystreptomycin, for instance, showed a 160-fold increase in the $S / N$ ratio. Most of the sulfonamides, a group of relatively basic compounds, were detected using GCB. Sulfaphenazole adducts, however, were not detected using GCB, whereas all proton, sodium, and potassium adducts were detected using oxidized GCB. S/N ratios were 18, 22, and 23 for these adducts, respectively.

Both acidic and basic compounds were selected from the human medicines group for evaluation. Among this group, the basic medicines were detected using both GCB and oxidized GCB. The $\mathrm{S} / \mathrm{N}$ ratios were higher for nearly all adducts that were formed when oxidized GCB was used, but it was not possible to predict which adduct would increase in signal intensity. The effect was most pronounced for carbamazepine, which is a polycyclic alkaline drug. The detection limit achieved for propranolol desorbed from the surface of oxidized GCB is comparable to that for DIOS chip [27].

Oxidized GCB showed significant potential to enhance the $S / N$ ratio when it was used for desorption ionization MS analysis of polar aminoglycoside antibiotics. Only the sodium adducts of gentamycin and tobramycin were detected using GCB. At least one adduct of each compound was detected when oxidized GCB was used. Organophosphate esters were also desorbed and ionized from the surfaces of GCB and oxidized GCB. As shown, they were mostly detectable using GCB, although the $\mathrm{S} / \mathrm{N}$ ratios were improved up to 23-fold for tetraethyl ethylenediphosphonate when oxidized GCB was used.

As shown in Table 1 in the supplementary material the reproducibility of $\mathrm{S} / \mathrm{N}$ values was improved when oxidized GCB was used, most likely attributable to more homogeneous spots, which made it more suitable for quantitative analysis.

\section{Quantitative Determination of Propranolol in Mussels}

The quantitative determination of propranolol in mussels, exposed to the compound as part of an ecotoxicological study, was performed to further evaluate the performance of the oxidized GCB material. Propranolol was extracted from mussels as described in the experimental section. The extract from mussels living in water containing propranolol $(100 \mu \mathrm{g} / \mathrm{L})$ was spotted onto both GCB and oxidized GCB and analyzed by means of SALDI. As shown in Figure 4, propranolol could be detected only by using oxidized GCB.

To determine the concentration of propranolol in mussels, a calibration curve was plotted of relative response (i.e., response for the analyte divided by that for the internal standard) against analyte concentration. Deuterated propranolol was used as the internal standard. Every point on the calibration curve represented an average of five spectra and each spectrum was accumulated from 10 laser shots at five different spots (i.e., a total of 50 laser shots). The linear response range for propranolol was in the region $0.1-20 \mu \mathrm{g} / \mathrm{mL}$, the $\mathrm{R}^{2}$ value was 0.9986 , and the RSD value for each point on the curve was $<10 \%$.

The concentration of propranolol extracted from mussels kept in water containing propranolol at 100 $\mu \mathrm{g} / \mathrm{L}$ was measured in five replicate samples and was found to be $1.02 \pm 0.11 \mu \mathrm{g} / \mathrm{g}$ wet weight of mussel, based on the protonated propranolol. This corresponds to a 10-fold bioaccumulation of propranolol by Baltic Sea blue mussels (Mytilus edulus). The recovery of

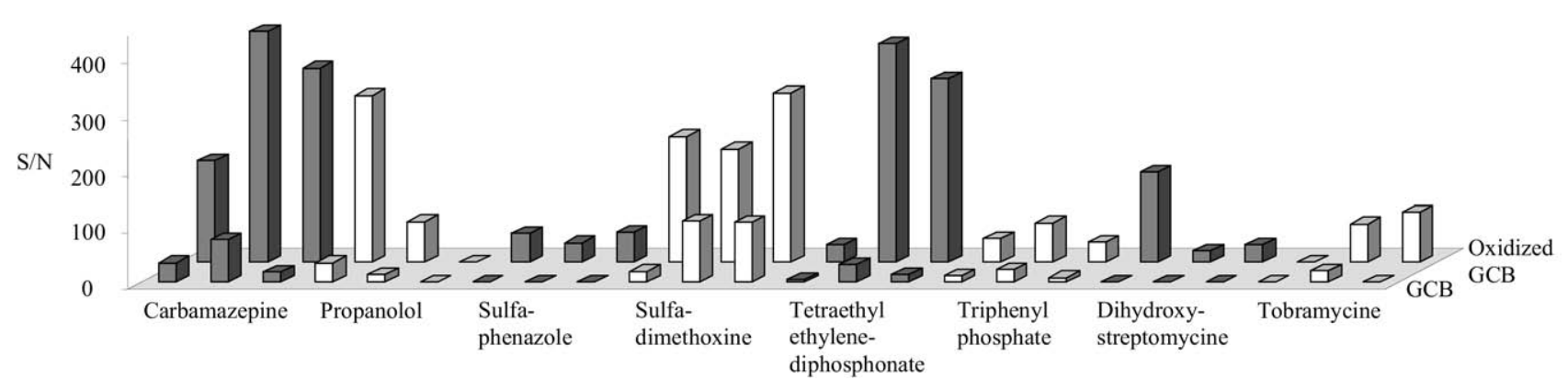

Figure 3. A comparison of signal-to-noise ratios (S/N) obtained using GCB and oxidized GCB as SALDI surfaces for the analysis of selected human medicines, sulfonamides, organophosphate esters, and aminoglycoside antibiotics. For each compound, the left bar depicts $\mathrm{S} / \mathrm{N}$ of $[\mathrm{M}+\mathrm{H}]^{+}$, the middle bar $\mathrm{S} / \mathrm{N}$ of $[\mathrm{M}+\mathrm{Na}]^{+}$, and the right bar $\mathrm{S} / \mathrm{N}$ of $[\mathrm{M}+\mathrm{K}]^{+}$. 

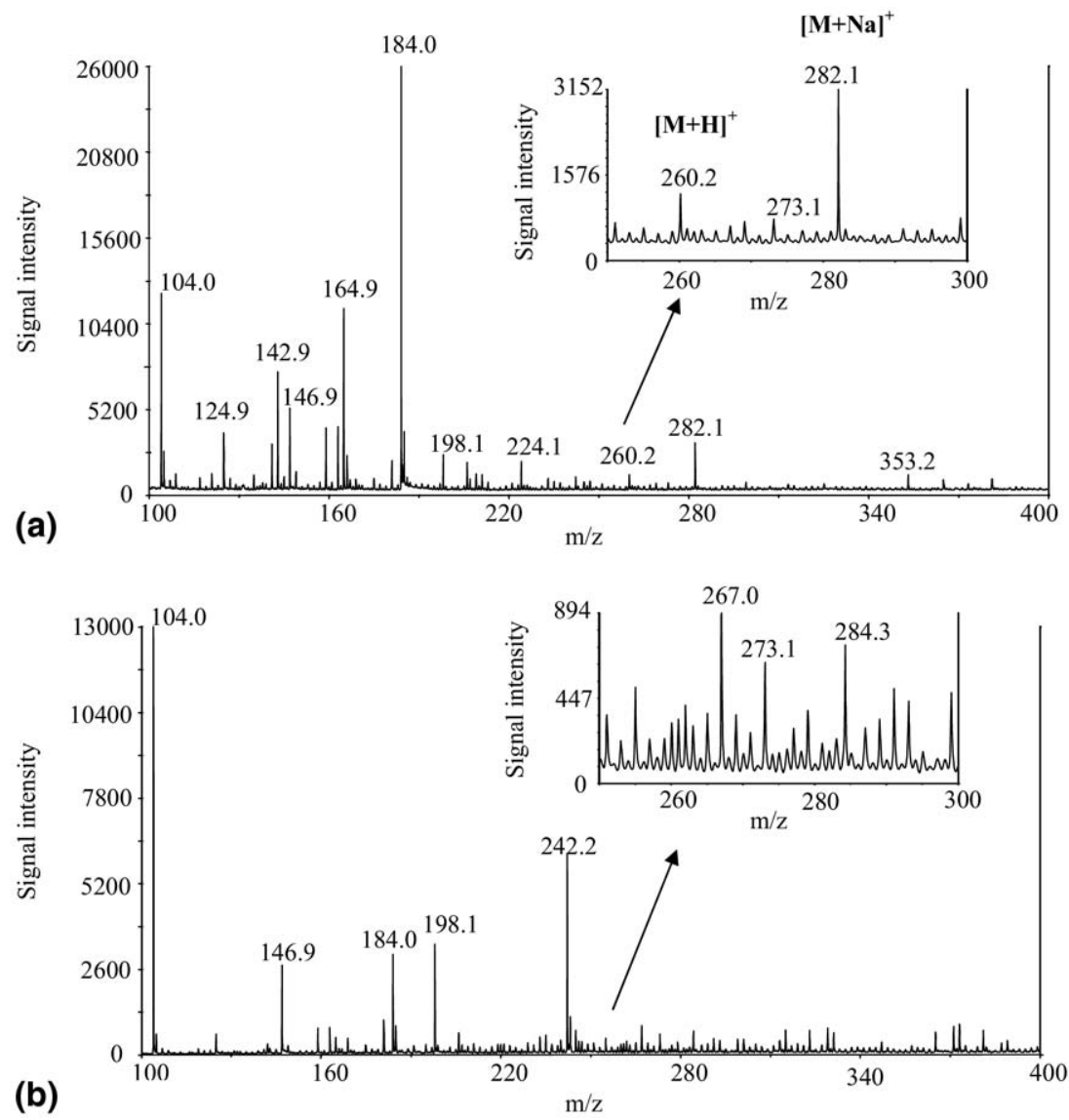

Figure 4. Mass spectra of propranolol extracted from Baltic Sea blue mussel living in water containing propanolol at $100 \mu \mathrm{g} / \mathrm{mL}$, spotted on (a) oxidized GCB and (b) GCB. The $\mathrm{m} / z$ values of 260 and 282 corresponding to $[\mathrm{M}+\mathrm{H}]^{+}$and $[\mathrm{M}+\mathrm{Na}]^{+}$, respectively, were detectable only when oxidized GCB was used as the SALDI surface.

propranolol from spiked blank mussels was also evaluated and found to be $59.8 \pm 3.7 \%$ for four replicates.

To detect $[\mathrm{M}+\mathrm{H}]^{+}$and $[\mathrm{M}+\mathrm{Na}]^{+}$the concentration of propranolol must be greater than 176 and 66 $\mathrm{ng} / \mathrm{g}$ wet weight of mussel, respectively, using a S/N ratio of 3 to define the limit of detection.

\section{Conclusions}

The SEM and titration experiments performed were used to quantify the extent of carboxylic groups on the surface of oxidized GCB particles. Proton NMR spectra acquired in $\mathrm{D}_{2} \mathrm{O}$ and DMSO confirmed the presence of the carboxylic groups. The addition of carboxylic groups to the surface of GCB not only increased the ionization yields but also changed the hydrophobicity of the surface, as demonstrated by the alteration in desorption profiles of QAs. The $\mathrm{S} / \mathrm{N}$ ratios of a wide number of analytes, and especially basic compounds, were significantly increased when oxidized GCB particles were used as the SALDI surface.

\section{Acknowledgments}

Dr. Linda Kumblad and Hanna Ericson at the Department of Systems Ecology, Stockholm University, are gratefully acknowledged for providing the samples of exposed mussels. The authors also thank Dr. Sahar Nikkhou Aski at the Department of Physical Chemistry, Stockholm University for her help with the NMR data, Kjell Jansson at the Department of Inorganic Chemistry, Stockholm University for performing SEM, and the Stockholm University Proteomics Facility (SUPF) for the use of MALDI.

\section{Appendix A Supplementary Material}

Supplementary material associated with this article can be found in the online version, at 10.1016/j.jasms.2009. 02.017 .

\section{References}

1. Zhong, G.; Lin, H. A Binary Matrix for Background Suppression in MALDI-MS of Small Molecules. Anal. Bioanal. Chem. 2007, 387, 19391944.

2. Cohen, L. H.; Gusev, A. I. Small Molecule Analysis by MALDI Mass Spectrometry. Anal. Bioanal. Chem. 2002, 373, 571-586.

3. Wei, J.; Buriak, J. M.; Siuzdak, G. Desorption-Ionization Mass Spectrometry on Porous Silicon. Nature 1999, 399, 243-246. 
4. Pepeterson, D. S.; Luo, Q.; Hilder, E. F.; Svec, F.; Frechet, J. M. J. Porous Polymer Monolith for Surface-Enhanced Laser Desorption/Ionization Time-of-Flight Mass Spectrometry of Small Molecules. Rapid Commun. Mass Spectrom. 2004, 18, 1504-1512.

5. Kinumi, T.; Saisu, T.; Takayama, M.; Niwa, H. Matrix-Assisted Laser Desorption/Ionization Time-of-Flight Mass Spectrometry Using an Inorganic Particle Matrix for Small Molecule Analysis. J. Mass Spectrom. 2000, 35, 417-422.

6. Ayorinde, F. O.; Hambright, P.; Porter, T. N.; Keith, Q. L., Jr. Use of meso-Tetrakis(pentafluorophenyl)porphyrin as a Matrix for Low Molecular Weight Alkylphenol Ethoxylates in Laser Desorption/Ionization Time-of-Flight Mass Spectrometry. Rapid Commun. Mass Spectrom. 1999, $13,2474-2479$

7. Grant, D. C.; Helleur, R. J. Surfactant-Mediated Matrix-Assisted Laser Desorption/Ionization Time-of-Flight Mass Spectrometry of Small Molecules. Rapid Commun. Mass Spectrom. 2007, 21, 837-845.

8. Pan, C.; Xu, S.; Hu, L.; Su, X.; Ou, J.; Zou, H.; Guo, Z.; Zhang, Y.; Guo, B. Using Oxidized Carbon Nanotubes as Matrix for Analysis of Small Molecules by MALDI-TOF MS. J. Am. Soc. Mass Spectrom. 2005, 16, 883-892.

9. Chen, Y.-C.; Shiea, J.; Sunner, J. Rapid Determination of Trace Nitrophenolic Organics in Water by Combining Solid-Phase Extraction with Surface-Assisted Laser Desorption/Ionization Time-of-Flight Mass Spectrometry. Rapid Commun. Mass Spectrom. 2000, 14, 86-90.

10. Sunner, J.; Dratz, E.; Chen, Y.-C. Graphite Surface-Assisted Laser Desorption/Ionization Time-of-Flight Mass Spectrometry of Peptides and Proteins from Liquid Solutions. Anal. Chem. 1995, 67, 4335-4342.

11. Shariatgorji, M.; Amini, N.; Thorsen, G.; Crescenzi, C.; Ilag, L. L. $\mu$-Trap for the SALDI-MS Screening of Organic Compounds Prior to LC/MS Analysis. Anal. Chem. 2008, 80, 5515-5523.

12. Lewis, W. G.; Shen, Z.; Finn, M. G.; Siuzdak, G. Desorption/ionization on Silicon (DIOS) Mass Spectrometry: Background and Applications. Int. J. Mass Spectrom. 2003, 226, 107-116.

13. Rawel, H. M.; Rohn, S.; Kroll, J.; Schweigert, F. J. Surface-Enhanced Laser Desorptions Ionization-Time of Flight-Mass Spectrometry Analysis in Complex Food and Biological Systems. Mol. Nutr. Food Res. 2005, $49,1104-1111$.

14. Choma, J.; Burakiewicz-Mortka, W.; Jaroniec, M.; Li, Z.; Klinik, J. Monitoring Changes in Surface and Structural Properties of Porous Carbons Modified by Different Oxidizing Agents. J. Colloid Interface Sci. 1999, 214, 438-446.
15. Papirer, E.; Dentzer, J.; Li, S.; Doonet, J. B. Surface Groups on Nitric Acid Oxidized Carbon Black Samples Determined by Chemical and Thermodesorption Analyses. Carbon 1991, 29, 69-72.

16. Rasheed, A.; Howe, J. Y.; Dadmun, M. D.; Britt, P. F. The Efficiency of the Oxidation of Carbon Nanofibers with Various Oxidizing Agents. Carbon 2007, 45, 1072-1080.

17. Campanella, L.; Di Corcia, A.; Samperi, R.; Gambacorta, A. The Nature of Surface Chemical Heterogeneities of Graphitized Carbon Black. Mater. Chem. 1982, 7, 429-438.

18. Richardson, S. D. Water Analysis: Emerging Contaminants and Curren Issues. Anal. Chem. 2007, 79, 4295-4324.

19. Halling-Sörensen, B.; Nors Nielsen, S. Lanzky, P. F. Ingerslev, F. Holten Lützhøft, H. C.; Jørgensen, S. E. Occurrence, Fate and Effects of Pharmaceuticals in the Environment: A Review. Chemosphere 1998, 36 357-393.

20. Löffler, D.; Römbke, J.; Meller, M.; Ternes, T. A. Environmental Fate of Pharmaceuticals in Water/Sediment Systems. Environ. Sci. Technol. 2005, 39, 5209-5218.

21. Diaz-Cruz, M. S.; de Alda, M. J. L.; Barcelo, D. Environmental Behavior and Analysis of Veterinary and Human Drugs in Soils, Sediments and Sludge. Trends Anal. Chem. 2003, 22, 340-351.

22. Hu, H.; Bhowmik, P.; Zhao, B.; Hamon, M. A.; Itkis, M. E.; Haddon, R. C. Determination of the Acidic Sites of Purified Single-Walled Carbon Nanotubes by Acid-Base Titration. Chem. Phys. Lett. 2001, 345, 25-28.

23. Bayne, B. L. Effects of Stress and Pollution on Marine Animals; Praeger: UK, 1985.

24. Kitaygorodskiy, A.; Wang, W.; Xie, S.-Y.; Lin, Y.; Fernando, K. A. S. Wang, X.; Qu, L.; Chen, B.; Sun, Y.-P. NMR Detection of Single-Walled Carbon Nanotubes in Solution. J. Am. Chem. Soc. 2005, 127, 7517-7520.

25. Pawsey, S.; McCormick, M.; De Paul, S.; Graf, R.; Lee, Y. S.; Reven, L.; Spiess, H. W. ${ }^{1} \mathrm{H}$ Fast MAS NMR Studies of Hydrogen-Bonding Interactions in Self-Assembled Monolayers. J. Am. Chem. Soc. 2003, 125 4174-4184

26. Harris, R. K.; Jackson, P.; Merwin, L. H.; Say, B. J. Perspectives in High-Resolution Solid-State Nuclear Magnetic Resonance, with Emphasis on Combined Rotation and Multiple-Pulse Spectroscopy. J. Chem. Soc. Faraday Trans. 1 1988, 84, 3649-3672.

27. Nordström, A.; Apon, J. V.; Uritboonthai, W.; Go, E. P.; Siuzdak, G. Surfactant-Enhanced Desorption/Ionization on Silicon Mass Spectrometry. Anal. Chem. 2006, 78, 272-278. 